\title{
Ages and metallicities of early-type galaxies
}

\author{
Ricardo Ogando ${ }^{1,2}$, Marcio Maia ${ }^{1}$, Paulo Pellegrini ${ }^{1}$ and Luiz da \\ Costa $^{1}$ \\ ${ }^{1}$ Grupo de Pesquisas em Astronomia, Observatório Nacional, \\ 20921-400, Rio de Janeiro, Brazil \\ email: ogando,maia,pssp,ldacosta@on.br \\ ${ }^{2}$ Instituto de Física, UFRJ, \\ Box 68528, Rio de Janeiro, Brazil (former PhD student)
}

\begin{abstract}
The study of stellar populations in early-type galaxies give us clues on how they form and evolve. We calculate age, $[\mathrm{Z} / \mathrm{H}]$, and $[\alpha / \mathrm{Fe}]$ ratio for 162 early-type galaxies using the SSP models from Thomas, Maraston, \& Bender (2003) applied to Lick indices measurements, such as $\mathrm{H} \beta, \mathrm{Mgb}, \mathrm{Fe} 5270$ and Fe5335. Those were obtained from longslit spectra observed in the ESO $1.52 \mathrm{~m}$ telescope as described in Ogando et al. (2008). We study the relations between the SSP parameters and velocity dispersion, as well as the influence of environment on these relations. We find that age, $[\mathrm{Z} / \mathrm{H}]$, and $[\alpha / \mathrm{Fe}]$ correlate well with velocity dispersion, so that more massive galaxies, have on average, higher metallicities, ages and abundance ratios than that of the low-mass ones. Galaxies in high density regions are older and more metal-rich than those in regions with low number of neighbors. These results are not consistent with standard predictions of hierarchical clustering. In the last decade, this "anti-hierarchical" behavior has also been generally tagged as downsizing and has challenged the current theoretical framework of galaxy formation, calling for new ways of star formation regulation in early-type galaxies.
\end{abstract}

Keywords. galaxies, stellar populations

The formation and evolution of early-type galaxies are fundamental to our understanding of the Universe. The traditional hierarchical clustering (HC) scenario predicts a longer assembly and formation time for massive galaxies (Kauffmann et al. 1993), so one should expect them to be younger than low-mass galaxies. However, the opposite is often observed, where low-mass early-type galaxies show signs of a young stellar population (Trager et al. 2000a, Sánchez-Blázquez et al. 2006b), particularly those in low-density environments, while massive galaxies tend to present a certain degree of maturity, specially in high-density regions. In fact, the observation of mature and massive galaxies beyond half-way the age of the Universe (Cimatti et al. 2004), and extended star formation in small objects (Cowie et al. 1996) introduced a new paradigm known as downsizing.

The ENEAR survey is this work data source and has the following characteristics: $|b|>30, \mathrm{~m}_{B}=14.5$ and $v_{r} \leqslant 7000 \mathrm{kms}^{-1}$. A detailed description of the sample is presented in Ogando et al. (2008). With respect to environmental analysis, we use the HyperLeda catalog, complete up to $\mathrm{m}_{B}=15.5$, to find companions according to the following rules: $\Delta v_{r}<750 \mathrm{kms}^{-1}$, projected separation $<500 \mathrm{kpc}$, companion has $\mathrm{M}_{B}<$ -16 . Given the number of companions, we define three environments: low $(\mathrm{N}=4, \mathrm{LD})$, medium $(4<\mathrm{N}<22, \mathrm{MD})$ and high density ( $\mathrm{N}=22, \mathrm{HD})$. In Ogando et al. (2008), for 509 galaxies it was measured the velocity dispersion and 10 Lick indices $(\mathrm{H} \beta$, Fe5015, $\mathrm{Mg}_{1}, \mathrm{Mg}_{2}, \mathrm{Mgb}, \mathrm{Fe} 5270$, Fe5335, Fe5406, Fe5709, NaD). From that sample we measure SSP parameters (age, $[\mathrm{Z} / \mathrm{H}]$ and $[\alpha / \mathrm{Fe}]$ ) for 162 galaxies that fell inside the model grids by Thomas, Maraston, \& Bender (2003), using the following indices: $\mathrm{H} \beta$, Mgb, Fe5270, Fe5335. Details of the SSP properties fitting procedure will be presented in 


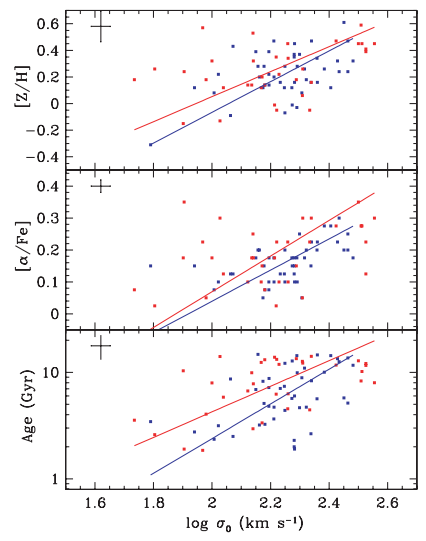

Figure 1. $[\mathrm{Z} / \mathrm{H}],[\alpha / \mathrm{Fe}]$ and age as a function of $\sigma_{0}$ for early-type galaxies separated in environments. The red (blue) points represent objects of high (low) density region. The continuous lines represent the respective bisector fit.

Ogando et al. (2010). Briefly, the SSP parameters for each galaxy are produced by a $\chi^{2}$ fit between the data and an interpolation of the model grid. The distribution of SSP parameters for the entire sample has average values of $[\mathrm{Z} / \mathrm{H}]=0.213 \pm 0.180$, Age $=9.1 \pm 4.3 \mathrm{Gyr}$, and $[\alpha / \mathrm{Fe}]=0.168 \pm 0.083$.

We investigate how the SSP parameters behave with velocity dispersion $\left(\log \sigma_{0}\right)$ as a function of environment. In Fig. 1 we omit the medium density objects and focus in the dichotomy between low (blue dots) and high (red dots) density environment. Instead of ordinary least-square fits we show bisector fits. We use the latter as a tentative to take into account the effects of the grids boundaries applied to the data. However, both fits give similar results with respect to the comparison between environs. We reproduce wellknown results from the literature, where massive early-type galaxies are old, metal-rich and had a short star formation history (SFH). Furthermore, there is a trend to high density environs to enhance this effect, which agrees with hierarchical clustering models De Lucia et al. (2006). Also, low mass galaxies seem to have their SFH truncated as they are older in HD. Thus, we reinforce the findings of Ogando et al. (2008). Massive galaxies are older, more metal-rich and had shorter SFH than low-mass galaxies, which favour the scenario known as downsizing. Furthermore, environmental effects can be more important to low-mass galaxies star formation history than to that of massive ones.

\section{Acknowledgement}

RO, MM, PSSP and LdC acknowledge CNPq for fellowships and grants.

\section{References}

Cimatti, A., Daddi, E., Renzini, A. et al. 2004, Nature, 430, 184

Cowie, L. L., Songaila, A., Hu, E. M., \& Cohen, J. G. 1996, AJ, 112, 839

De Lucia, G., Springel, V., White, S. D. M. et al. 2006, $M N R A S, 366,499$

Kauffmann, G., White, S. D. M., \& Guiderdoni, B. 1993, MNRAS, 264, 201

Ogando, R. L. C., Maia, M. A. G., Pellegrini, P. S., \& da Costa, L. N. 2008, AJ, 135, 2424

Ogando, R. L. C., Maia, M. A. G., Pellegrini, P. S., \& da Costa, L. N. 2010, in preparation

Thomas, D., Maraston, C., \& Bender, R. 2003, MNRAS, 339, 897

Trager, S. C., Faber, S. M., Worthey, G., \& González, J. J. 2000a, AJ, 119, 1645

Sánchez-Blázquez, P., Gorgas, J., Cardiel, N. et al. 2006b, A\&̈A, 457, 809 